\title{
THERMOELASTIC CONTACT PROBLEMS WITH RADIATION BOUNDARY CONDITIONS
}

\author{
By G. M. L. GLADWELL \\ (Solid Mechanics Division, University of Waterloo, Waterloo, Ontario, \\ Canada N2L 3G1) \\ and J. R. BARBER \\ (Department of Mechanical Engineering and Applied Mechanics, University \\ of Michigan, Ann Arbor, Michigan 48109, U.S.A.)
}

[Received 16 June 1982]

\section{SUMMAR Y}

Thermoelastic contact problems are usually analysed with the idealised thermal boundary conditions of perfect conduction or perfect insulation. This paper makes use of some recently derived results for thermal problems of the half-space under radiation boundary conditions to solve two thermoelastic problems for a heated rigid punch identing an elastic half-space. In the first it is supposed that there is a thermal resistance between punch and half-space and the remainder of the boundary is insulated. In the second, the punch is in perfect thermal contact while the remainder of the boundary is partially insulated. Numerical results and graphs are presented for both problems.

\section{Introduction}

THE problem of the semi-infinite elastic solid indented by a rigid cylindrical heated punch under steady-state thermal conditions was first treated by George and Sneddon (1) and Olesiak (2). In both papers, as in most subsequent work in this field, it was assumed that either the temperature or the heat flux was prescribed at any given point on the surface of the half-space and hence the determination of the temperature field was reduced to a classical boundary-value problem of potential theory. For example, if the punch is a perfect conductor at temperature $T_{0}$ in good thermal contact with the half-space $z>0$ over the circle $0 \leqslant r \leqslant a, z=0$, whilst the rest of the surface is insulated, the temperature $T$ is determined from the equations

and

$$
\begin{gathered}
T=T_{0}, \quad 0 \leqslant r \leqslant a, \quad z=0, \\
\frac{\partial T}{\partial z}=0, \quad r>a, \quad z=0,
\end{gathered}
$$

It is clear that these boundary conditions are at best idealisations of the physical problem, since there will always be some heat lost from the exposed [Q. J Mech appl. Math, Val. 36, Pt. 3, 1983] 
surface and some resistance to heat flow from the punch into the half-space. A more realistic boundary condition is the radiation condition, defined for example by Carslaw and Jaeger (3), according to which the heat flux into the half-space,

$$
q=-k \frac{\partial T}{\partial z},
$$

where $k$ is the conductivity of the half-space, is proportional to the difference between the local surface temperature and that of a surrounding medium. With this condition, we have

$$
-k \frac{\partial T}{\partial z}=h\left(T_{0}-T\right), \quad z=0,
$$

where $T_{0}$ is here the prescribed temperature of the medium and $h$ is a constant coefficient of surface heat transfer. This equation can be cast in dimensionless form by writing $z=\zeta a, r=\rho a$, where $a$ is the punch radius, giving

$$
\frac{\partial T}{\partial \zeta}=H\left(T-T_{0}\right),
$$

where $H=h a / k$ is a dimensionless surface heat-transfer coefficient, sometimes known as a Biot number (4).

\section{Statement of the problems}

In this paper, we shall consider two thermoelastic contact problems involving boundary conditions of the form of equation (1.5), making use of a recent solution to boundary-value problems of this kind (5).

\subsection{The heated punch with thermal contact resistance (problem I)}

A flat cylindrical rigid punch at temperature $T_{0}$ is pressed into the surface of the half-space $\zeta>0$ with a force $P$, but the flow of heat across the interface is obstructed by a constant contact resistance $1 / h_{1}$ per unit area. The exposed surface is taken to be insulated, giving the boundary conditions

$$
\begin{aligned}
& \frac{\partial T}{\partial \zeta}-H_{1} T=-H_{1} T_{0}, \quad 0 \leqslant \rho \leqslant 1, \quad \zeta=0, \\
& \frac{\partial T}{\partial \zeta}=0, \quad \rho>1, \quad \zeta=0,
\end{aligned}
$$

where $H_{1}=h_{1} a / k$. The contact is assumed to be frictionless so that the mechanical boundary conditions are

$$
\begin{aligned}
\sigma_{r z} & =0, \quad \rho \geqslant 0, \quad \zeta=0, \\
w & =\text { constant }, \quad 0 \leqslant \rho \leqslant 1, \quad \zeta=0, \\
\sigma_{z z} & =0, \quad \rho>1, \quad \zeta=0,
\end{aligned}
$$




$$
2 \pi a^{2} \int_{0}^{1} \rho \sigma_{z x} d \rho=-P,
$$

where $\sigma_{r z}, \sigma_{z z}$ are the shear and normal stress, respectively, and $w$ is the normal surface displacement.

\subsection{Radiation at the exposed surface (problem II)}

For this problem, we postulate good thermal contact between the punch and the surface of the half-space in $0 \leqslant \rho \leqslant 1$, but allow radiation into a medium at zero temperature in $\rho>1$, giving the thermal boundary conditions

$$
\begin{gathered}
T=T_{0}, \quad 0 \leqslant \rho \leqslant 1, \quad \zeta=0, \\
\frac{\partial T}{\partial \zeta}-H_{2} T=0, \quad \rho>1, \quad \zeta=0,
\end{gathered}
$$

the mechanical conditions being again given by equations (2.3) to (2.6).

These two problems may be regarded as the limiting cases $H_{2} \rightarrow 0$ (in problem I), $H_{1} \rightarrow \infty$ (in problem II) of the general problem with radiation through different coefficients $H_{1}, H_{2}$ in the regions $0 \leqslant \rho \leqslant 1, \rho>1$, respectively. In practice we should expect heat transfer through the contact to be much easier than that into the atmosphere outside the contact area, i.e., $H_{1} \gg H_{2}$. If this is so and if the dimensionless quantity $H_{1}$ is of order unity, then $\mathrm{H}_{2}$ will tend to zero (problem I); similarly if $\mathrm{H}_{2}$ is of order unity then $H_{1}$ will tend to infinity (problem II). Equations (1.1), (1.2), of course, correspond to the more restrictive case in which $H_{2} \rightarrow 0$ and $H_{1} \rightarrow \infty$.

\section{Solution of the contact problem}

The contact problem can be reduced to a mixed boundary-value problem in potential theory, using a solution of the equations of thermoelastic equilibrium in terms of harmonic potential functions. A sufficiently general representation for frictionless contact problems is

$$
\begin{gathered}
\mathbf{a} / a=4(1-\nu) \mathbf{k} \frac{\partial \psi}{\partial \zeta}-2(1-\nu) \nabla \psi+(1-2 \nu) \nabla \omega+\zeta \nabla \frac{\partial \omega}{\partial \zeta}-(3-4 \nu) \mathbf{k} \frac{\partial \omega}{\partial \zeta}, \\
T=\frac{2(1-\nu)}{\alpha(1+\nu)} \frac{\partial^{2} \psi}{\partial \zeta^{2}}
\end{gathered}
$$

where $\mathbf{n}$ is the displacement vector, $\nabla^{2} \psi=\nabla^{2} \omega=0$, and $\mu, \nu, \alpha$ are, respectively, the shear modulus, Poisson's ratio and coefficient of thermal expansion for the material of the half-space. This is solution $B$ of Barber 
(6). On the surface $\zeta=0$, we have

$$
\begin{gathered}
w=2(1-\nu) a\left(\frac{\partial \psi}{\partial \zeta}-\frac{\partial \omega}{\partial \zeta}\right), \\
\sigma_{z z}=-2 \mu \frac{\partial^{2} \omega}{\partial \zeta^{2}}, \\
T=\frac{2(1-\nu)}{\alpha(1+\nu)} \frac{\partial^{2} \psi}{\partial \zeta^{2}}, \\
q=-\frac{k}{a} \frac{\partial T}{\partial \zeta}=-\frac{2 k(1-\nu)}{\alpha(1+\nu) a} \frac{\partial^{3} \psi}{\partial \zeta^{3}} .
\end{gathered}
$$

The thermal and mechanical problems are uncoupled in that $T$ and hence $\psi$ can be determined from the thermal boundary conditions ( $(2.1)$ and (2.2) in problem I, (2.7), (2.8) in problem II) without reference to the mechanical problem. Once $\psi$ is known, the mechanical conditions (2.4) to (2.5) define a boundary-value problem for $\omega$. Thus

$$
\begin{array}{cc}
\frac{\partial \omega}{\partial \zeta}=\frac{\partial \psi}{\partial \zeta}+B, & 0 \leqslant \rho \leqslant 1, \quad \zeta=0, \\
\frac{\partial^{2} \omega}{\partial \zeta^{2}}=0, & \rho>1, \quad \zeta=0,
\end{array}
$$

where $B$ is a constant related to the displacement of the punch.

\section{A note on the rigid-body displacement}

In principle, we have the option of specifying either the compressive load $P$ applied to the punch (equation (2.6)) or its rigid-body displacement and hence the constant $B$ in equation (3.7). However, difficulties arise with the second formulation unless there is no net heat influx to the body, i.e., unless

$$
\int_{0}^{\infty} \rho \frac{\partial T}{\partial \zeta} d \rho=0, \quad \zeta=0
$$

If this condition is not satisfied, the temperature and hence the thermal strains will behave as $O(1 / \rho)$ at large values of $\rho$ and the displacements will be logarithmically unbounded. A similar difficulty, of course, arises in two-dimensional isothermal problems for the half-plane and it is essentially a consequence of the assumed infinite extent of the solid. The use of such solutions depends on the implicit assumption that quantities which are found to be regular at infinity will approximate the correct local behaviour in the corresponding problem for a finite body, provided the contact area is small compared with the dimensions of the body. The above discussion shows that displacement is not such a quantity except under condition (4.1). 
In the problems described here, this difficulty will be encountered in determining $\partial \psi / \partial \zeta$ for substitution into equations (3.7). A suitably regular solution will be found for $T$ and hence $\partial^{2} \psi / \partial \zeta^{2}$, and the most natural way to proceed is to integrate the relation

$$
\frac{1}{\rho} \frac{\partial}{\partial \rho}\left\{\rho \frac{\partial}{\partial \rho}\left(\frac{\partial \psi}{\partial \zeta}\right)\right\}=-\frac{\partial^{3} \psi}{\partial \zeta^{3}}
$$

on $\zeta=0$, which follows from $\partial \psi / \partial \zeta$ being harmonic. However, if condition (4.1) is not satisfied, it will prove impossible to find an integral of equation (4.2) which is regular both at zero and infinity.

An alternative approach, not susceptible to this difficulty is to write

$$
\omega=\omega_{1}+\omega_{2},
$$

where $\omega_{1}, \omega_{2}$ satisfy the boundary conditions

$$
\begin{array}{ccc}
\frac{\partial \omega_{1}}{\partial \zeta}=C, & 0 \leqslant \rho \leqslant 1, & \zeta=0, \\
\frac{\partial^{2} \omega_{1}}{\partial \zeta^{2}}=0, & \rho>1, & \zeta=0, \\
\frac{\partial^{3} \omega_{2}}{\partial \zeta^{3}}=\frac{\partial^{3} \psi}{\partial \zeta^{3}}, & 0 \leqslant \rho \leqslant 1, & \zeta=0, \\
\frac{\partial^{2} \omega_{2}}{\partial \zeta^{2}}=0, & \rho>1, & \zeta=0 .
\end{array}
$$

If equations (4.6), (4.7) are treated as defining a classical mixed boundaryvalue problem for the harmonic function $\partial^{2} \omega_{2} / \partial \zeta^{2}$, the resulting solution will be continuous at $\rho=1, \zeta=0$. However, the necessary singularity in contact pressure will be contributed by $\omega_{1}$. The arbitrary constant $C$ will finally be calculated from equation (2.6) for the prescribed total load on the punch.

\section{The solution of problem I}

The thermal boundary conditions (2.1), (2.2) correspond to case (ii) of (5). Thus, using equations (2.1), (4.4), (4.5) of (5) we may write

$$
T(\rho, \zeta)=\mathscr{K}_{0}\left[\xi^{-1} A(\xi) \exp (-\xi \zeta) ; \rho\right]
$$

where

$$
A(\xi)=\int_{0}^{1} f(x) \cos (\xi x) d x
$$

and $f(x)$ satisfies the equation

$$
-\frac{1}{\rho} \frac{d}{d \rho} \int_{\rho}^{1} \frac{x f(x) d x}{\left(x^{2}-\rho^{2}\right)^{\frac{1}{2}}}+H \int_{0}^{\rho} \frac{f(x) d x}{\left(\rho^{2}-x^{2}\right)^{\frac{1}{2}}}=H T_{0},
$$


the approximate solution of which is discussed in (5). In terms of this representation, we have

$$
\frac{\partial^{3} \psi}{\partial \zeta^{3}}=\frac{\alpha(1+\nu)}{2(1-\nu)} \frac{\partial T}{\partial \zeta}=\frac{\alpha(1+\nu)}{2(1-\nu)} \frac{1}{\rho} \frac{d}{d \rho} \int_{\rho}^{1} \frac{x f(x) d x}{\left(x^{2}-\rho^{2}\right)^{\frac{1}{2}}}, \quad 0 \leqslant \rho \leqslant 1, \quad \zeta=0 .
$$

We now insert this into equations (4.6), (4.7) for $\partial^{2} \omega_{2} / \partial \zeta^{2}$, using the representation

$$
\frac{\partial^{2} \omega_{2}}{\partial \zeta^{2}}(\rho, \zeta)=\mathscr{K}_{0}\left[\xi^{-1} B(\xi) \exp (-\xi \zeta) ; \rho\right],
$$

with which equations (4.6), (4.7) become

$$
\begin{array}{cl}
\mathscr{K}_{0}[B(\xi) ; \rho]=-\partial^{3} \psi / \partial \zeta^{3}, & 0 \leqslant \rho \leqslant 1, \\
\mathscr{K}_{0}\left[\xi^{-1} B(\xi) ; \rho\right]=0, & \rho>1 .
\end{array}
$$

Operating on equation (5.7) by $\mathscr{A}_{2}^{-1}$, as in case (i) of (5), we find

$$
B(\xi)=-\int_{0}^{1} g(x) \sin (\xi x) d x,
$$

and

$$
\begin{gathered}
\frac{\partial^{2} \omega_{2}}{\partial \zeta^{2}}(\rho, 0)=-\int_{\rho}^{1} \frac{g(t) d t}{\left(t^{2}-\rho^{2}\right)^{\frac{1}{2}}}, \quad 0 \leqslant \rho \leqslant 1, \\
\frac{\partial^{3} \omega_{2}}{\partial \zeta^{3}}(\rho, 0)=\frac{1}{\rho} \frac{d}{d \rho} \int_{0}^{\min (\rho, 1)} \frac{\operatorname{tg}(t) d t}{\left(\rho^{2}-t^{2}\right)^{\frac{1}{2}}} .
\end{gathered}
$$

Thus, with equations (5.4), (5.10), equation (4.6) becomes

$$
\frac{1}{\rho} \frac{d}{d \rho} \int_{0}^{\rho} \frac{\operatorname{tg}(t) d t}{\left(\rho^{2}-t^{2}\right)^{\frac{1}{2}}}=\frac{\alpha(1+\nu)}{2(1-\nu)} \frac{1}{\rho} \frac{d}{d \rho} \int_{\rho}^{1} \frac{x f(x) d x}{\left(x^{2}-\rho^{2}\right)^{\frac{1}{2}}}, \quad 0 \leqslant \rho \leqslant 1 .
$$

Integrating both sides we have

$$
\int_{0}^{\rho} \frac{\operatorname{tg}(t) d t}{\left(\rho^{2}-t^{2}\right)^{\frac{1}{2}}}=\frac{\alpha(1+\nu)}{2(1-\nu)}\left\{\int_{\rho}^{1} \frac{x f(x) d x}{\left(x^{2}-\rho^{2}\right)^{\frac{1}{1}}}-\int_{0}^{1} f(x) d x\right\},
$$

which is an Abel integral equation for $g(t)$ with solution ((2.27) of (5))

$$
g(x)=\frac{\alpha(1+\nu)}{\pi(1-\nu)} \frac{1}{x} \frac{d}{d x}\left[\int_{0}^{x} \frac{t}{\left(x^{2}-t^{2}\right)^{\frac{1}{1}}}\left\{\int_{t}^{1} \frac{s f(s) d s}{\left(s^{2}-t^{2}\right)^{\frac{1}{2}}}-\int_{0}^{1} f(s) d s\right\} d t\right] .
$$

After some manipulations, this reduces to

$$
g(x)=\frac{\alpha(1+\nu) x}{\pi(1-\nu)} \int_{0}^{1} \frac{f(s) d s}{s^{2}-x^{2}}, \quad 0 \leqslant x \leqslant 1 .
$$


To complete the solution we have to solve for $\omega_{1}$ and determine the constant $C$ using equation (2.6). The conditions on $\omega_{1}$ are of a well-known form and, on $\zeta=0$,

$$
\frac{\partial^{2} \omega_{1}}{\partial \zeta^{2}}=-\frac{C}{\left(1-\rho^{2}\right)^{\frac{1}{2}}}, \quad 0 \leqslant \rho<1 .
$$

The contact pressure can now be obtained from equation (3.4) as

$$
\begin{aligned}
p(\rho) & =-\sigma_{z z}(\rho, 0)=2 \mu \frac{\partial^{2} \omega}{\partial \zeta^{2}} \\
& =-2 \mu\left\{\frac{C}{\left(1-\rho^{2}\right)^{\frac{1}{2}}}+\int_{\rho}^{1} \frac{g(t) d t}{\left(t^{2}-\rho^{2}\right)^{\frac{1}{2}}}\right\},
\end{aligned}
$$

whilst the total load is

$$
\begin{aligned}
P & =2 \pi a^{2} \int_{0}^{1} \rho p(\rho) d \rho \\
& =-4 \mu \pi a^{2}\left\{C+\int_{0}^{1} \operatorname{tg}(t) d t\right\},
\end{aligned}
$$

after a reversal of the order in the second term. Since $P$ is a prescribed quantity and $g(t)$ is completely known through equation (5.14), equation (5.17) can be considered as an equation for the constant $C$.

For computational purposes it is convenient to use the expansion (4.6) of (5), namely

$$
f(x)=T_{0} \sum_{n=0}^{\infty} a_{n} \sin (2 n+1) \theta, \quad x=\cos \theta
$$

then (5.14) yields

$$
g(x)=-\frac{\alpha(1+\nu)}{2(1-\nu)} T_{0} \sum_{n=0}^{\infty} a_{n} \cos (2 n+1) \theta
$$

so that

$$
P=-4 \pi \mu a^{2} C-2 \pi \gamma T_{0} a^{2} \sum_{n=0}^{\infty} \frac{a_{n}}{(2 n-1)(2 n+3)},
$$

where

$$
\gamma=\mu \alpha(1+\nu) /(1-\nu) .
$$

In order to compute $p(\rho)$ we use (3.8), (3.9) of (5) and write

$$
\sum_{n=0}^{\infty} a_{n} \cos (2 n+1) \theta=\frac{1}{\sin \theta} \sum_{n=0}^{\infty} a_{n}^{*} \cos (2 n+1) \theta, \quad 0 \leqslant \theta<\frac{1}{2} \pi,
$$


where

$$
a_{n}^{*}=\sum_{m=0}^{\infty} c_{m, n} a_{m},
$$

and $c_{m, n}$ is given by (3.17) of (5). Then equation (5.16) gives

$$
p(\rho)=-\frac{2 \mu C}{\left(1-\rho^{2}\right)^{\frac{1}{2}}}+\frac{\gamma \pi}{2} T_{0} \sum_{n=0}^{\infty} a_{n}^{*} P_{n}\left(2 \rho^{2}-1\right) .
$$

The first term in equation (5.16) clearly dominates the pressure distribution near $\rho=1$ and hence there will be inadmissable tensile stresses if $C>0$. The limiting load, $P_{+}$, which is just sufficient to prevent this condition is given by the second term of equation (5.20), namely

$$
P_{+}=-2 \pi \gamma T_{0} a^{2} \sum_{n=0}^{\infty} \frac{a_{n}}{(2 n-1)(2 n+3)} .
$$

In the physical problem, if $P<P_{+}$, separation will occur near $\rho=1$.

If $T_{0}<0$, i.e., if the punch is cooled, the second term in equation (5.16) defines a tensile contact stress with a maximum value at the centre $(\rho=0)$, and the solution is physically meaningful only if the first term is sufficiently large to leave $p(0)>0$ (see (7) for a discussion of this matter in the limiting case $H \rightarrow \infty$ ). This requires $P \rightarrow P_{-}$, where

$$
\begin{gathered}
P_{-}=P_{+}-2 \pi \gamma T_{0} a^{2} \sum_{n=0}^{\infty} h_{n} a_{n}, \\
h_{n}=\int_{0}^{\frac{1}{2 \pi}} \frac{\cos (2 n+1) \theta \sin \theta d \theta}{\cos \theta}=\frac{\pi}{4}\left\{2(-1)^{n}-c_{n}\right\},
\end{gathered}
$$

and $c_{n}$ is given by (5.26) of (5).

Figure 1 shows the variation of dimensionless contact pressure

$$
p^{*}(\rho)=p(\rho) /\left(\gamma T_{0}\right)
$$

with radius $\rho$ for $H=1$ and various values of dimensionless load ratio

$$
P^{*}=\pi P /\left(4 \gamma T_{0} a^{2}\right) .
$$

The upper portion relates to $T_{0}>0$ and $P^{*}=\beta P_{+}^{*}$, where $\beta \geqslant 1$, and $\beta=1$ corresponds to the onset of tensile stress at the outer boundary $\rho=1$. Similarly, the lower portion corresponds to $T_{0}<0$ and $P^{*}=\beta P^{*}$ so that $\beta \geqslant 1$ and $\beta=1$ corresponds to the onset of tensile stress at the centre, $\rho=0$. The values of $P_{+}^{*}$ and $P_{-}^{*}$ are plotted against $H$ in Fig. 2 .

It may be verified that in the limiting case $H \rightarrow \infty$,

$$
\begin{gathered}
f(x)=\frac{2 T_{0}}{\pi}, \quad g(x)=\frac{\alpha(1+\nu)}{\pi^{2}(1-\nu)} T_{0} \ln \left|\frac{1-x}{1+x}\right|, \\
P_{+}^{*}=1, \quad P_{-}^{*}=1-\frac{1}{4} \pi^{2},
\end{gathered}
$$




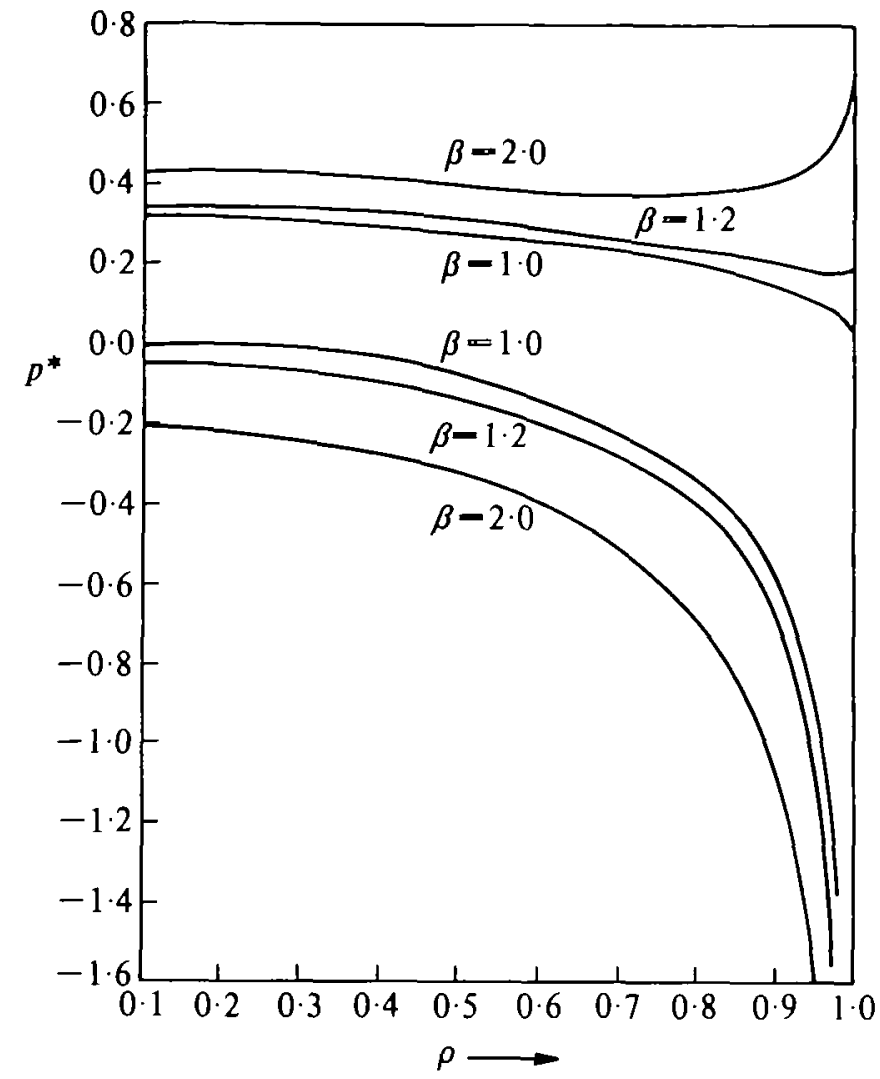

FIG. 1. The variation of dimensionless pressure with radius.

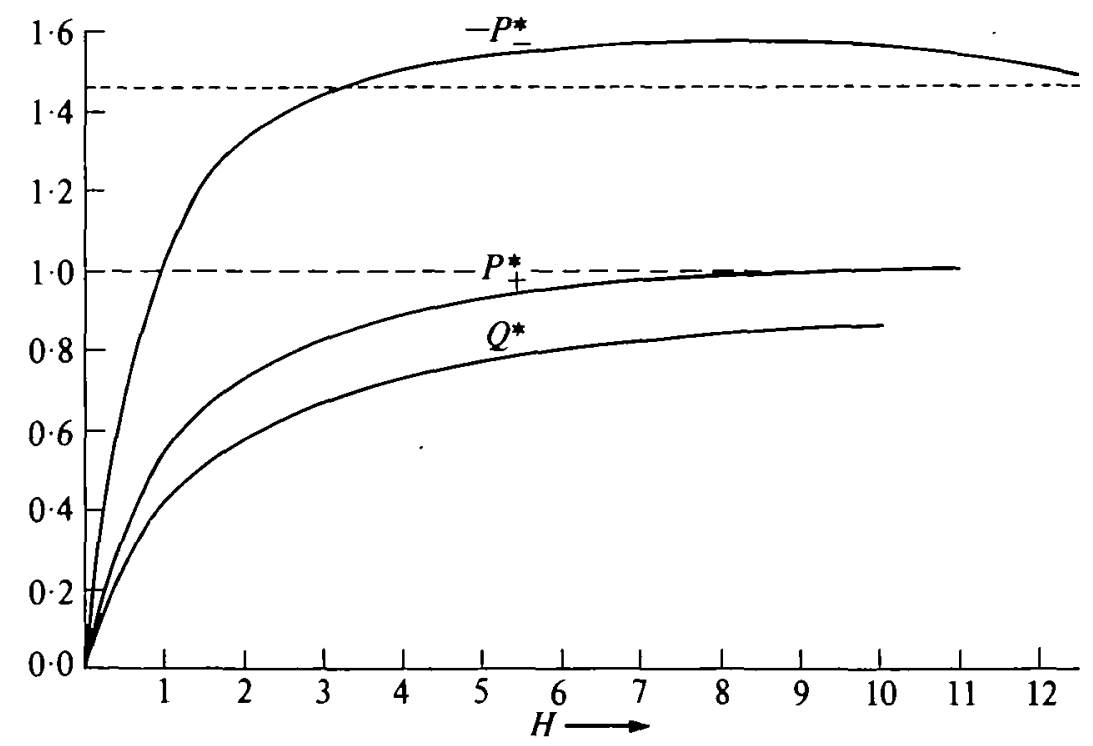

FIG. 2. The variation of dimensionless critical loads and total heat flux with $H$ in problem $I$. 
while for small $H$

$$
\begin{array}{cc}
f(x)=\frac{2 T_{0} H}{\pi}\left(1-x^{2}\right)^{\frac{1}{2}}, & g(x)=-\frac{\alpha(1+\nu)}{\pi(1-\nu)} T_{0} H x, \\
P_{+}^{*}=\frac{1}{3} \pi H, & P_{-}^{*}=-\frac{2}{3} \pi H .
\end{array}
$$

It is also of interest to calculate the effect of the thermal contact resistance on the heat-flux distribution $q$ over the contact area, and on the total heat flux $Q$. From equations (3.6) and (5.4) we have

$$
q=-\frac{k}{a} \frac{\partial T}{\partial \zeta}=-\frac{k}{a \rho} \frac{d}{d \rho} \int_{\rho}^{1} \frac{t f(t) d t}{\left(t^{2}-\rho^{2}\right)^{\frac{1}{2}}}, \quad 0<\rho<1,
$$

and, when the representation (5.18) is used, we find

$$
q=\frac{\pi k}{2 a} T_{0} \sum_{n=0}^{\infty}(2 n+1) a_{n} P_{n}\left(2 \rho^{2}-1\right) .
$$

The total heat flux is

$$
Q=2 \pi a^{2} \int_{0}^{1} \rho q(\rho) d \rho=2 \pi a k \int_{0}^{1} f(t) d t=\frac{1}{2} \pi^{2} a k T_{0} a_{0} .
$$

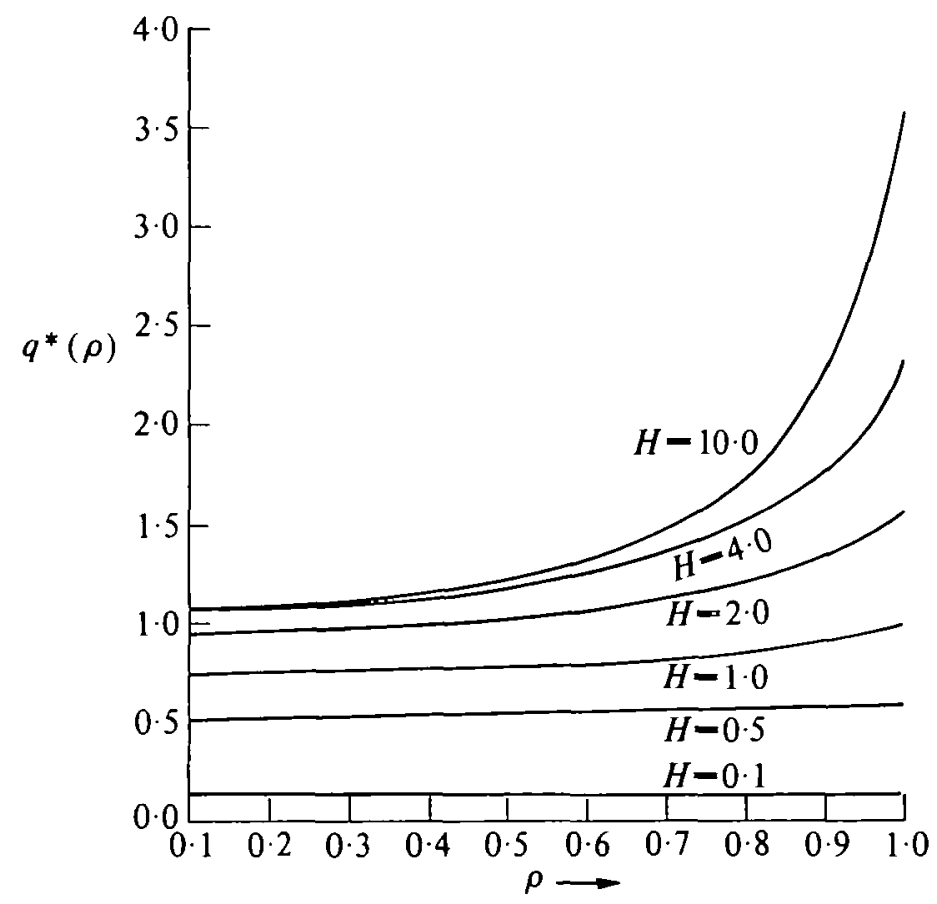

FIG. 3. The variation of heat flux with radius for various values of $\boldsymbol{H}$. 
The dimensionless total heat flux,

$$
Q^{*}=Q /\left(4 a k T_{0}\right)
$$

is plotted against $H$ in Fig. 2, and typical distributions of

$$
q^{*}(\rho)=\pi a q /\left(2 k T_{0}\right)
$$

are shown in Fig. 3. The heat-flux distribution shows peak values near $\rho=1$ when $H$ is large and these tend to

$$
q^{*}(\rho)=\left(1-\rho^{2}\right)^{-\frac{1}{2}}, \quad Q^{*}=1,
$$

in the limit $H \rightarrow \infty$ (perfect contact). At small values of $H$ a uniform heat flux is approached, with

$$
q^{*}=\frac{1}{2} \pi H, \quad Q^{*}=\frac{1}{4} \pi H .
$$

\section{The solution of problem II}

We now consider the contact problem with radiation in the external region defined by equations (2.7), (2.8). These equations are considered in case (iii) of (5), and the thermal field $T$ is written as $T=T_{1}+T_{2}+T_{3}$, where $T_{3}$ is expressed in terms of the solution $f(x)$ of

$$
\frac{1}{\rho} \frac{d}{d \rho} \int_{\rho}^{\infty} \frac{x f(x) d x}{\left(x^{2}-\rho^{2}\right)^{\frac{1}{2}}}-H \int_{1}^{\rho} \frac{f(x) d x}{\left(\rho^{2}-x^{2}\right)^{\frac{1}{2}}}=R(\rho)
$$

with

$$
R(\rho)=\frac{2 T_{0} H}{\pi}\left\{\sin ^{-1}\left(\frac{1}{\rho}\right)-\frac{\left(\rho^{2}-1\right)^{\frac{1}{2}}}{\rho^{2}}\right\}-\frac{T_{0}}{\rho^{3}}
$$

With $f(x)$ determined, we can proceed as in problem $I$ to find $\partial^{2} \omega_{2} / \partial \zeta^{2}$ from the conditions

$$
\begin{gathered}
\frac{\partial^{3} \omega_{2}}{\partial \zeta^{3}}=\frac{\partial^{3} \psi}{\partial \zeta^{3}}=\frac{\alpha(1+\nu)}{2(1-\nu)}\left(\frac{\partial T_{1}}{\partial \zeta}+\frac{\partial T_{2}}{\partial \zeta}+\frac{\partial T_{3}}{\partial \zeta}\right), \quad \rho<1 \\
\frac{\partial^{2} \omega_{2}}{\partial \zeta^{2}}=0, \quad \rho>1
\end{gathered}
$$

Using the representation

$$
\frac{\partial^{2} \omega_{2}}{\partial \zeta^{2}}=\mathscr{K}_{\mathrm{o}}\left[\xi^{-1} B(\xi) \exp (-\xi \zeta) ; \rho\right]
$$

we find

$$
B(\xi)=-\int_{0}^{1} g(x) \sin (\xi x) d x
$$


so that

$$
\frac{\partial^{3} \omega_{2}}{\partial \zeta^{3}}=\frac{1}{\rho} \frac{d}{d \rho} \int_{0}^{\rho} \frac{\operatorname{tg}(t) d t}{\left(\rho^{2}-t^{2}\right)^{\frac{1}{2}}}=\frac{\alpha(1+\nu)}{2(1-\nu)}\left(\frac{\partial T_{1}}{\partial \zeta}+\frac{\partial T_{2}}{\partial \zeta}+\frac{\partial T_{3}}{\partial \zeta}\right), \quad \rho<1 .
$$

Equations (5.5), (5.7), and (5.12) of (5) show that

$$
\frac{\partial T}{\partial \zeta}=-\frac{2 T_{0}}{\pi}\left(1-\rho^{2}\right)^{-\frac{1}{2}}+\frac{2 T_{0}}{\pi \rho^{3}}\left\{\sin ^{-1} \rho-\rho\left(1-\rho^{2}\right)^{-\frac{1}{1}}\right\}+\frac{1}{\rho} \frac{d}{d \rho} \int_{1}^{\infty} \frac{x f(x) d x}{\left(x^{2}-\rho^{2}\right)^{\frac{1}{2}}},
$$

so that, on integration

$$
\int_{0}^{\rho} \frac{\operatorname{tg}(t) d t}{\left(\rho^{2}-t^{2}\right)^{\frac{1}{2}}}=\frac{\alpha(1+\nu)}{2(1-\nu)}\left[\frac{2 T_{0}}{\pi}\left\{\left(1-\rho^{2}\right)^{\frac{1}{2}}-\frac{\sin ^{-1} \rho}{\rho}\right\}+\int_{1}^{\infty} \frac{x f(x) d x}{\left(x^{2}-\rho^{2}\right)^{\frac{1}{2}}}-\int_{1}^{\infty} f(x) d x\right] .
$$

Solving for $g(t)$ we have $\dagger$

$g(t)=\frac{\alpha(1+\nu)}{2(1-\nu)} \frac{2}{\pi}\left[\frac{2 T_{0}}{\pi}\left\{\frac{1}{t}-\frac{1}{2} \ln \left(\frac{1+t}{1-t}\right)-\frac{1}{2 t^{2}} \ln \left(\frac{1+t}{1-t}\right)\right\}+t \int_{1}^{\infty} \frac{f(s) d s}{s^{2}-t^{2}}\right]$.

Expressions for the pressure distribution $p(\rho)$ and the total load $P$ can be found, as in problem I, from equations (5.16), (5.17). The total load is

$$
P=-4 \pi \mu a^{2} C+\pi \gamma a^{2}\left\{\left(1-\frac{4}{\pi^{2}}\right) T_{0}-\frac{2}{\pi} \int_{1}^{\infty} s f(s) \ln \left(\frac{s+1}{s-1}\right) d s\right\} .
$$

The condition of no tensile stress requires

$$
P>P_{+}, \quad T_{0}>0, \quad P<P_{-}, \quad T_{0}<0,
$$

where

$$
\begin{gathered}
P_{+}=\pi \gamma a^{2}\left\{\left(1-\frac{4}{\pi^{2}}\right) T_{0}-\frac{2}{\pi} \int_{1}^{\infty} s f(s) \ln \left(\frac{s+1}{s-1}\right) d s\right\}, \\
P_{-}=\pi \gamma a^{2}\left\{-\frac{8}{\pi^{2}} T_{0}+\frac{1}{\pi} \int_{1}^{\infty}\left(1-s^{2}\right) f(s) \ln \left(\frac{s+1}{s-1}\right) d s\right\} .
\end{gathered}
$$

These quantities may be expressed in terms of the $\beta_{n}$-coefficients in equation (5.18) of (5); they are

$$
\begin{gathered}
P_{+}=\pi \gamma T_{0} a^{2}\left\{\left(1-\frac{4}{\pi^{2}}\right)-4 \sum_{n=1}^{\infty} k_{n} \beta_{n}\right\}, \\
P_{-}=\pi \gamma T_{0} a^{2}\left\{-\frac{8}{\pi^{2}}-4 \sum_{n=1}^{\infty} k_{n} \beta_{n}+\sum_{n=1}^{\infty} \beta_{n}\left(\frac{1}{2 n-1}+\frac{1}{2 n+1}\right)\right\},
\end{gathered}
$$

† The last term in equation (6.9) is zero when $H \neq 0$, as shown in (5); it is retained in the derivation of equation (6.10) as this leads to a simpler final expression. 


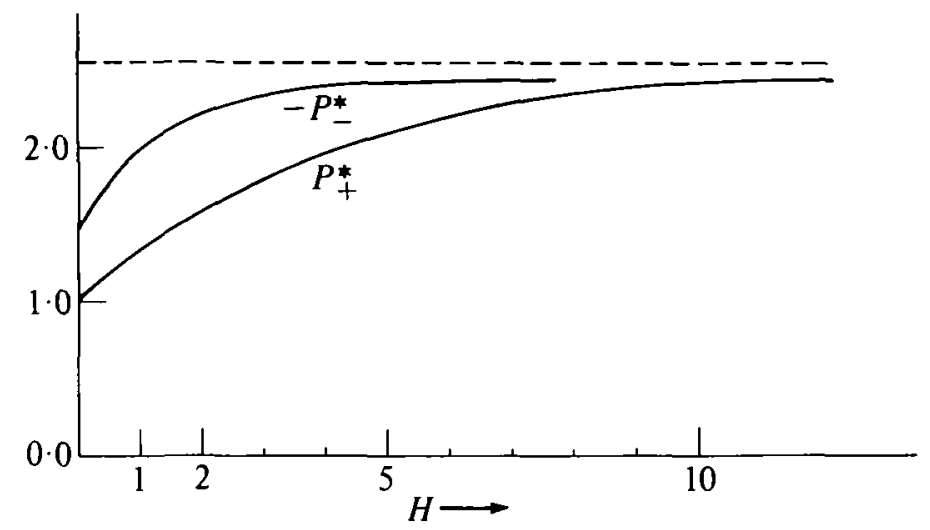

FIG. 4. The variation of dimensionless critical loads with $H$ in problem II.

where $k_{n}$ is given by

$$
k_{n}=\sum_{m=1}^{\infty}(-1)^{m-1} /(2 n+2 m-1) .
$$

Figure 4 shows $P_{+}^{*}$ and $P_{-}^{*}$ as functions of $H$. The limiting values of $P_{+}^{*}$ and $P_{-}^{*}$ are

$$
\begin{array}{cccc}
P_{+}^{*}=1, & P_{-}^{*}=1-\frac{1}{4} \pi^{2} & \text { when } & H=0, \\
P_{+}^{*} \rightarrow \frac{1}{4} \pi^{2}, & P_{-}^{*} \rightarrow-\frac{1}{4} \pi^{2} & \text { when } & H \rightarrow \infty .
\end{array}
$$

The rigid-body displacement of the punch relative to the point at infinity is bounded for this problem, and can be calculated from the relation

$$
\begin{aligned}
\frac{1}{\rho} \frac{\partial}{\partial \rho}\left(\rho \frac{\partial w}{\partial \rho}\right) & =-2(1-\nu) a\left(\frac{\partial^{3} \psi}{\partial \zeta^{3}}-\frac{\partial^{3} \omega_{2}}{\partial \zeta^{3}}\right) \\
& =-\alpha(1+\nu) a\left(\frac{\partial T_{1}}{\partial \zeta}+\frac{\partial T_{2}}{\partial \zeta}+\frac{\partial T_{3}}{\partial \zeta}\right)+2(1-\nu) a \frac{\partial^{3} \omega}{\partial \zeta^{3}}
\end{aligned}
$$

derived from (3.3), (3.5) and the fact that $\partial \psi / \partial \zeta, \partial \omega / \partial \zeta$ are harmonic. The term involving $\omega_{1}$ has been omitted since it merely introduces an additive constant into the final expression for punch displacement. Now using equations (5.3), (5.7), (5.10) of (5) and (6.5) we find that on $\zeta=0$

$$
\begin{aligned}
\frac{1}{\rho} \frac{d}{d \rho}\left(\rho \frac{d w}{d \rho}\right)= & -\alpha(1+\nu) a\left\{\frac{T_{0}}{\rho^{3}}+\frac{1}{\rho} \frac{d}{d \rho} \int_{\rho}^{\infty} \frac{x f(x) d x}{\left(x^{2}-\rho^{2}\right)^{\frac{1}{1}}}\right\}+ \\
& +2(1-\nu) a \frac{1}{\rho} \frac{d}{d \rho} \int_{0}^{1} \frac{x g(x) d x}{\left(\rho^{2}-x^{2}\right)^{\frac{1}{2}}}, \quad \rho>1 .
\end{aligned}
$$


Assuming that $\rho(d w / d \rho) \rightarrow 0$ as $\rho \rightarrow \infty$ (as it must if $w$ is to be bounded) we integrate twice throughout to obtain

$$
\begin{aligned}
w \equiv w(1)-w(\infty)= & \alpha(1+\nu) a\left\{-T_{0}+\int_{1}^{\infty} \frac{d \rho}{\rho} \int_{\rho}^{\infty} \frac{x f(x) d x}{\left(x^{2}-\rho^{2}\right)^{\frac{1}{2}}}\right\} \\
& -2(1-\nu) a \int_{1}^{\infty} \frac{d \rho}{\rho} \int_{0}^{1} \frac{x g(x) d x}{\left(\rho^{2}-x^{2}\right)^{\frac{1}{2}}}
\end{aligned}
$$

Carrying out some of the integrations, we find

$$
w=\alpha(1+\nu) a\left\{-T_{0}+\int_{1}^{\infty} \cosh ^{-1} x f(x) d x\right\}-2(1-\nu) a \int_{0}^{1} \sin ^{-1} x g(x) d x \text {. }
$$

Now substituting for $g(x)$ from equation (6.10) and carrying out more integrations we obtain

$$
\begin{aligned}
w & =\alpha(1+\nu) T_{0} a\left\{-\frac{2}{\pi}+\frac{1}{2} \int_{1}^{\infty} \ln \left(s^{2}-1\right) f(s) d s\right\}, \\
& =-\alpha(1+\nu) T_{0} a\left(\frac{2}{\pi}+\frac{\pi}{2} \sum_{n=1}^{\infty} \frac{\beta_{2 n-1}}{2 n-1}\right) .
\end{aligned}
$$

This expression defines the rigid-body displacement of the punch until the load $P$ takes the limiting value $P_{+}$.

The displacement $w$ increases without limit as $H \rightarrow 0$ and it is therefore tempting to attribute the infinite rigid-body displacement in previous thermoelastic contact solutions to the physically unrealistic nature of the insulated external boundary condition. However, it should be noted that equation (6.21) is only slowly convergent when $H$ is small, in other words, the punch displacement depends significantly upon the thermal strains at a large distance from the contact area. Hence, if the solution is applied to a finite body, the usual requirement that $L \gg a$, where $L$ is a specimen dimension of the body, will not generally be sufficient to ensure accurate results for punch displacement. The more stringent condition $H L \gg a$ must also be satisfied. However, the weaker condition is sufficient so far as calculations of stress and temperature fields are concerned and, if $H$ is sufficiently small, these quantities converge on the insulated boundary solution. Thus, there is a significant range of problems for which the simpler solution gives as much information as can be obtained from a half-space representation of the body.

\section{Acknowledgement}

The impetus for this research came when one of the authors (J. R. Barber) was visiting the other at the University of Waterloo under a grant from the National Science and Engineering Research Council of Canada. The computations were carried out by Rikk Marejka. 


\section{REFERENCES}

1. D. L. George and I. N. Sneddon, J. Math. Mech. 11 (1962) 665.

2. Z. Olesiak, Bull. de l'Acad. Pol. Sci. 13 (1965) 439.

3. H. S. Carslan and J. C. Jaeger, Conduction of Heat in Solids (Clarendon Press, Oxford, 1959).

4. W. H. McAdams, Heat Transmission (McGraw-Hill, New York, 1954) 135.

5. G. M. L. Gladwell, J. R. Barber and Z. Olesiak, $Q$. $I$ Mech. appl. Math. 36 (1983) 387

6. J. R. Barber, in A. D. de PATER and J. J. KALker (Editors), The Mechanics of the Contact Between Deformable Bodies (Delft University Press, 1975) 177.

7. - Int. J. Mech. Sci: 15 (1973) 813. 